\title{
EARLY-WARNING MODEL OF INFLUENZA A VIRUS PANDEMIC BASED ON PRINCIPAL COMPONENT ANALYSIS
}

\author{
GAO, J. ${ }^{1,2, *}-$ XU, H. X. ${ }^{1}-$ DING, T. ${ }^{1}-$ WANG, K. ${ }^{1}$ \\ ${ }^{1}$ School of science, Jiangnan University, Wuxi 214122, China \\ (phone/ fax: +86-510-85910532) \\ ${ }^{2}$ Key Laboratory of Systems Biology, Shanghai Institutes for Biological Sciences, Chinese \\ Academy of Sciences, Shanghai 200031, China \\ (phone/fax: +86-21-64365937) \\ *Corresponding author \\ e-mail: gaojie@jiangnan.edu.cn; phone:+86-510-85912033
}

(Received 28 ${ }^{\text {th }}$ Oct 2016; accepted $28^{\text {th }}$ Feb 2017)

\begin{abstract}
During the courses of the human history, several major influenza pandemics caused great disasters to human beings. In this paper, we choose all PA protein sequences of influenza A virus from 1933 to 2013, and these PA protein sequences are translated into chaos game representation walk sequences. For each CGR walk sequence we calculate 25 index values related to sequence structure characteristics, and principal component analysis method is used to study these values. Then we construct a principal component model to early-warn influenza A virus pandemic, compute the comprehensive index values CIV, and sort these values in descending order. Through computing the comprehensive index values based on the model and sorting, the comprehensive index value in 2009 is 30.274, ranked first; the comprehensive index value in 1969 is 8.383, ranked second; and the comprehensive index value in 1959 is 5.684, ranked third. And there were well-known influenza A virus pandemics in 2009, 1969, 1959. It is found that those influenza A virus pandemic years are almost at the top of the list, so we can draw a conclusion using the model that there maybe an influenza A virus pandemic when the CIV in the year is significantly bigger than that in the nearby years and the CIV in the year is more than 3.
\end{abstract}

Keywords: PA protein sequences, chaos game representation (CGR) walk model, PCA, eigenvalue, comprehensive index value (CIV)

\section{Introduction}

Influenza $\mathrm{A}$ is an acute respiratory infection. It is a disease with a fast transmission and may cause high morbidity and mortality in the world (Kobasa et al., 2004; Morens et al., 2004). In recent years, with the further spread of the flu, the World Health Organization has raised the flu alert level to its sixth grade (Sokolov et al., 2012). This has led many experts and scholars from all over the world to study the influenza from different aspects, and search for the methods to forecast influenza A virus pandemic. For example, Gao et al. (2013) found that a T160A mutation was identified at the 150-loop in the HA gene by means of real-time reverse-transcriptase-polymerase-chain-reaction assays, viral culturing and sequence analysis for clinical, epidemiologic, and virologic data from those patients. Pu et al. (2015) conclude that the prevalence and variation of H9N2 influenza 
virus in farmed poultry could provide an important early-warning of the emergence of novel reassortants with pandemic potential.

In 1990, chaos game representation (CGR) for DNA sequences has been proposed by Jeffrey (1990). In 2004, Yu et al proposed a new CGR algorithm of protein sequences based on the detailed hydrophobic-hydrophilic (HP) model (Yu et al., 2003, 2010). In 2009, Gao and Xu proposed a chaos game representation (CGR) walk model based on the new CGR coordinates for the protein sequences from complete genomes. In 2009, Scheffer et al. found that before the critical transition, some complex system such as ecological system, financial market and climate will show the general characteristics, such as leading to the increasing of variance and autocorrelation. In 2011, Ren and Gao used the variance of the influenza virus data as the early-warning signal to analyze and predict the pandemic year of influenza.

We choose all protein sequences of influenza A virus from 1933 to 2013, and the result of PA protein sequences is the most significant. These PA protein sequences are translated into CGR walk sequences. For each CGR walk sequence we calculate 25 index values related to sequence structure characteristics, and principal component analysis (PCA) method is used to study these values. Then we construct a principal component model to early-warn influenza A virus pandemic, compute the comprehensive index values (CIV), and sort these values in descending order. We will establish a warning system to forecast outbreak of influenza A pandemic.

\section{Material and Methods}

\section{Dataset}

There are 10 proteins PB2, PB1, PA, HA, NP, NA, M1, M2, NS1, NS2 in influenza A virus. Polymerase composed by protein $\mathrm{PA}$ and $\mathrm{PB} 1$ and $\mathrm{PB} 2$ often decides the difficulty of the virus infected host. So we analyze protein PA and PB1 and PB2, and find that the characteristic information of PA protein is the most obvious. And before 1933, there are less than 5 PA protein sequences of influenza A virus only in 1902, 1918, 1927, 1931. So we select all the influenza A virus PA protein sequences from 1933 to 2013. (data from the NCBI website: http://www.ncbi.nlm.nih.gov/genomes/FLU/Database/nph-select.cgi?go=database).

\section{CGR walk model}

In 1990 Jeffrey proposed CGR for DNA sequence. The CGR has been extended to protein sequence (Fisher et al., 1994; Basu et al., 1997).

For a given protein sequence $s=s_{1} s_{2} \cdots s_{n}$ with length n, where $s_{i}$ is one of the 20 kinds of amino acids for $i=1, \cdots, n$, we define 


$$
c_{i}=\left\{\begin{array}{cccccc}
A 0, & \text { if } & s_{i} & \text { is } & \text { non-polar, } & \\
A 1, & \text { if } & s_{i} & \text { is } & \text { negative } & \text { polar, } \\
A 2, & \text { if } & s_{i} & \text { is } & \text { uncharged } & \text { polar } \\
A 3, & \text { if } & s_{i} & \text { is } & \text { positive } & \text { polar },
\end{array}\right.
$$

and then obtain a sequence $X(s)=c_{1} c_{2} \cdots c_{n}$, where $c_{i}$ is a letter of an alphabet $\{A 0, A 1, A 2, A 3\}$. Next, we define a CGR for a sequence $X(s)$, similar to that of DNA sequence, in a square $[0,1] \times[0,1]$, where the four vertices correspond to the four letter $A 0, A 1, A 2$ and $A 3, A 0=(0,0), A 1=(0,1), A 2=(1,1), A 3=(1,0)$. The first point of the plot is placed half way between the center of the square and the vertex corresponding to the first letter of the sequence $X(s)$; the i-th point of the plot is then placed half way between the (i-1)th point and the vertex corresponding to the i-th letter

$$
C G R_{i}=C G R_{i-1}-0.5 \cdot\left(C G R_{i-1}-c_{i}\right), i=1, \ldots, n, C G R_{0}=(0.5,0.5)
$$

For a given protein sequence, we construct a CGR-walk model, where $y_{k}$ is the y-coordinate of $C G R_{k},{ }^{x}$ is the x-coordinate of $C G R_{k}$.

\section{PCA method}

PCA is a commonly used and effective multivariate statistic analysis method (Johnson and Wichern, 2001). Multiple original variables can be reduced into a few comprehensive indexes. Due to too many indexes and a certain correlation between each other, there is duplicate information in the observation data. It is difficult to study on the distribution of the sample in high dimension space. Therefore people want to substitute a few comprehensive indexes for plenty of original variables, and these comprehensive indexes contain enough information to reflect the original variables and they are independent each other.

Here we want to make comprehensive analysis and evaluation for the multiple indexes of protein data of influenza A virus each year using PCA method. The few comprehensive indexes can provide most of the information in the original indexes, therefore we can simplify the analysis of virus protein data. Finally we can make quantitative and comparison evaluation for each year's data. 


\section{Results}

\section{Indexes}

Based on the CGR-walk model for the protein sequence of influenza A virus, we can translated PA protein sequences into numerical sequences. Thus we can get the specific numerical data. Based on the principal of scientific and feasibility, we choose 25 indexes reflecting the sequence structure characteristic information of protein data of influenza $\mathrm{A}$ virus: average $\left(\mathrm{x}_{1}\right)$, variance $\left(\mathrm{x}_{2}\right)$, standard deviation $\left(\mathrm{x}_{3}\right)$, coefficient variation $\left(\mathrm{x}_{4}\right)$, lag $k$ autocorrelation coefficient $\left(\mathrm{x}_{\mathrm{k}}\right)$.

For each numerical sequence we calculate 25 index values.

\section{Data analysis based on PCA}

We compute the eigenvalue of correlation matrix, contribution and cumulative contribution of every principal component (Table 1).

According to Table 1, we can see that the contribution of the first principal component is $41.17 \%$, the contribution of the second principal component is $19.51 \%$, and the contribution of the third principal component is $10.11 \%$. This also shows that the three principal components have gathered about $70 \%$ data information of 25 indexes.

The selected principal component numbers should meet the general requirement that the cumulative contribution had better be greater than $85 \%$. We extract the first six principal components because their cumulative contribution has been up to $87.62 \%$ (Table 1). Therefore the first six principal components represent about $87.62 \%$ data information of all the original indexes.

Table 1. Eigenvalue, proportion and cumulative contribution of every principal component

\begin{tabular}{llll}
\hline item & eigenvalues & proportion & cumulative \\
\hline z1 & 9.88178292 & 0.4117 & 0.4117 \\
z2 & 4.68336852 & 0.1951 & 0.6069 \\
z3 & 2.42705361 & 0.1011 & 0.7080 \\
z4 & 1.62607717 & 0.0678 & 0.7758 \\
z5 & 1.42185141 & 0.0592 & 0.8350 \\
z6 & 0.98931913 & 0.0412 & 0.8762 \\
z7 & 0.77035157 & 0.0321 & 0.9083 \\
z8 & 0.48546446 & 0.0202 & 0.9286 \\
z9 & 0.46061874 & 0.0192 & 0.9477 \\
z10 & 0.29273349 & 0.0122 & 0.9599 \\
z11 & 0.24461422 & 0.0102 & 0.9701 \\
z12 & 0.13972687 & 0.0058 & 0.9760 \\
z13 & 0.10908996 & 0.0045 & 0.9805 \\
z14 & 0.09633166 & 0.0040 & 0.9845 \\
z15 & 0.07787623 & 0.0032 & 0.9878 \\
z16 & 0.06715905 & 0.0028 & 0.9906 \\
\hline
\end{tabular}




\begin{tabular}{llll}
\hline $\mathrm{z} 17$ & 0.05626723 & 0.0023 & 0.9929 \\
$\mathrm{z} 18$ & 0.04961278 & 0.0021 & 0.9950 \\
$\mathrm{z} 19$ & 0.03837153 & 0.0016 & 0.9966 \\
$\mathrm{z} 20$ & 0.02963200 & 0.0012 & 0.9978 \\
$\mathrm{z} 21$ & 0.02454026 & 0.0010 & 0.9988 \\
$\mathrm{z} 22$ & 0.01251669 & 0.0005 & 0.9993 \\
$\mathrm{z} 23$ & 0.00920517 & 0.0004 & 0.9997 \\
$\mathrm{z} 24$ & 0.00643533 & 0.0003 & 1.0000 \\
$\mathrm{z} 25$ & 0.00000000 & 0.0000 & 1.0000 \\
\hline
\end{tabular}

\section{Early-warning model of influenza A virus pandemic}

Here we use $x_{i}(i=1,2, \ldots, 25)$ as the 25 indexes, $z_{j}(j=1,2, \ldots, 6)$ as the first six principal components, $y$ as the CIV.

After normalizing the eigenvalues as the weights, we can construct a principal component model to make comprehensive analysis.

$$
y=0.470 z_{1}+0.223 z_{2}+0.115 z_{3}+0.077 z_{4}+0.068 z_{5}+0.047 z_{6}
$$

where the first six principal components are

$$
\begin{aligned}
& z_{1}=0.155 x_{1}+0.082 x_{2}+0.168 x_{3}-0.273 x_{5}-0.209 x_{6}+\ldots+0.110 x_{25} \\
& z_{2}=-0.346 x_{1}-0.147 x_{2}-0.200 x_{3}+0.158 x_{5}+0.181 x_{6}+\ldots-0.378 x_{25} \\
& z_{3}=0.214 x_{1}+0.129 x_{2}+0.159 x_{3}+0.120 x_{5}+0.119 x_{6}+\ldots+0.251 x_{25} \\
& z_{4}=0.007 x_{1}+0.0777 x_{2}+0.058 x_{3}+0.170 x_{5}+0.378 x_{6}+\ldots+0.018 x_{25} \\
& z_{5}=0.118 x_{1}+0.326 x_{2}+0.188 x_{3}+0.087 x_{5}+0.040 x_{6}+\ldots-0.037 x_{25} \\
& z_{6}=0.045 x_{1}+0.506 x_{2}+0.070 x_{3}+0.131 x_{5}+0.268 x_{6}+\ldots-0.004 x_{25}
\end{aligned}
$$

Then a principal component model is obtained, i.e.

$$
\begin{aligned}
& y=0.036 x_{1}+0.073 x_{2}+0.051 x_{3}-0.054 x_{5}+0.0002 x_{6}+0.119 x_{7}+0.095 x_{8}+0.156 x_{9}+ \\
& 0.161 x_{10}+0.166 x_{11}+0.037 x_{12}+0.099 x_{13}+0.155 x_{14}+0.051 x_{15}+0.155 x_{16}+0.066 x_{17}+ \\
& 0.091 x_{18}+0.129 x_{19}+0.137 x_{20}+0.095 x_{21}+0.153 x_{22}+0.121 x_{23}+0.090 x_{24}-0.005 x_{25}
\end{aligned}
$$


Through computing the CIVs based on the model and sorting, the CIV in 2009 is 30.274, ranked first; the CIV in 1969 is 8.383, ranked second; and the CIV in 1959 is 5.684, ranked third (Table 2).

Table 2. Principal component value, $C I V$ and ranking $(R)$ of every year

\begin{tabular}{|c|c|c|c|c|c|c|c|c|}
\hline year & $\mathrm{z} 1$ & $\mathrm{z} 2$ & $\mathrm{z} 3$ & $\mathrm{z} 4$ & $\mathrm{z} 5$ & z6 & CIV & $\mathrm{R}$ \\
\hline 2009 & 35.45261 & -64.3871 & 55.6356 & 32.82124 & 135.5943 & 208.8902 & 30.27 & 1 \\
\hline 1969 & 10.75625 & -20.3654 & 16.9396 & 9.437207 & 37.34962 & 56.40566 & 8.383 & 2 \\
\hline 1959 & 7.795853 & -15.1847 & 12.27423 & 6.59448 & 25.2774 & 37.55668 & 5.684 & 3 \\
\hline 2008 & 6.92281 & -13.5436 & 11.01679 & 5.961404 & 22.97595 & 34.28074 & 5.135 & 4 \\
\hline 2010 & 6.539319 & -12.886 & 10.38519 & 5.55199 & 21.17855 & 31.42996 & 4.741 & 5 \\
\hline 2011 & 6.425023 & -12.678 & 10.22931 & 5.473158 & 20.82713 & 30.89184 & 4.661 & 6 \\
\hline 1993 & 5.596687 & -11.1373 & 8.946197 & 4.743167 & 17.84305 & 26.32073 & 3.993 & 7 \\
\hline 1991 & 5.219861 & -10.5587 & 8.360391 & 4.335047 & 16.01551 & 23.41813 & 3.635 & 8 \\
\hline 1943 & 5.214887 & -10.4623 & 8.328537 & 4.348227 & 16.22068 & 23.80802 & 3.586 & 9 \\
\hline 1986 & 5.002644 & -10.0965 & 7.957286 & 4.099997 & 15.19687 & 22.18209 & 3.409 & 10 \\
\hline 2012 & 4.851384 & -9.89545 & 7.8407 & 4.053103 & 14.88543 & 21.75071 & 3.324 & 11 \\
\hline 1987 & 4.760841 & -9.71039 & 7.653763 & 3.932538 & 14.4574 & 21.0958 & 3.232 & 12 \\
\hline 1942 & 4.71931 & -9.66417 & 7.616887 & 3.931356 & 14.35521 & 20.91896 & 3.203 & 13 \\
\hline 1998 & 4.63469 & -9.38142 & 7.38231 & 3.786482 & 13.95378 & 20.32245 & 3.133 & 14 \\
\hline 1935 & 4.573606 & -9.384 & 7.399063 & 3.798539 & 13.84638 & 20.15095 & 3.091 & 15 \\
\hline 1984 & 4.525075 & -9.20382 & 7.21974 & 3.692189 & 13.56738 & 19.71275 & 3.040 & 16 \\
\hline 2006 & 4.443647 & -9.08068 & 7.137881 & 3.653652 & 13.3598 & 19.42347 & 2.989 & 17 \\
\hline 2004 & 4.408305 & -9.03353 & 7.096972 & 3.627309 & 13.22039 & 19.21263 & 2.979 & 18 \\
\hline 2007 & 4.398081 & -8.9953 & 7.084407 & 3.633615 & 13.27443 & 19.30528 & 2.968 & 19 \\
\hline 2000 & 4.394602 & -9.01406 & 7.095766 & 3.638541 & 13.24885 & 19.25685 & 2.960 & 20 \\
\hline 1990 & 4.384001 & -8.95316 & 7.07441 & 3.640189 & 13.33133 & 19.4291 & 2.957 & 21 \\
\hline 2013 & 4.338363 & -9.0209 & 7.109714 & 3.631746 & 13.14415 & 19.12288 & 2.919 & 22 \\
\hline 1980 & 4.312083 & -8.85831 & 6.949018 & 3.547294 & 12.87069 & 18.68706 & 2.879 & 23 \\
\hline 1940 & 4.278652 & -8.90727 & 7.025017 & 3.587957 & 12.96613 & 18.84638 & 2.878 & 24 \\
\hline 2001 & 4.260032 & -8.76218 & 6.873552 & 3.503521 & 12.69635 & 18.42958 & 2.843 & 25 \\
\hline 1981 & 4.232171 & -8.70129 & 6.848415 & 3.503136 & 12.72917 & 18.49092 & 2.840 & 26 \\
\hline 1989 & 4.222301 & -8.67173 & 6.787246 & 3.450174 & 12.53508 & 18.1458 & 2.804 & 27 \\
\hline 2002 & 4.196715 & -8.62875 & 6.764026 & 3.442491 & 12.49758 & 18.11223 & 2.794 & 28 \\
\hline 1982 & 4.187976 & -8.60569 & 6.735987 & 3.41699 & 12.45142 & 18.04004 & 2.784 & 29 \\
\hline 2003 & 4.111168 & -8.4786 & 6.667246 & 3.404529 & 12.32938 & 17.88454 & 2.751 & 30 \\
\hline 1966 & 4.110836 & -8.4605 & 6.650805 & 3.400187 & 12.26497 & 17.7928 & 2.744 & 31 \\
\hline 1946 & 4.065945 & -8.39225 & 6.574759 & 3.341303 & 12.05336 & 17.45467 & 2.712 & 32 \\
\hline 1988 & 4.063561 & -8.48662 & 6.676848 & 3.400406 & 12.21633 & 17.70217 & 2.695 & 33 \\
\hline 1999 & 4.037999 & -8.32806 & 6.518398 & 3.313335 & 11.96569 & 17.30743 & 2.675 & 34 \\
\hline 1985 & 3.997718 & -8.27397 & 6.472493 & 3.283428 & 11.82137 & 17.09198 & 2.640 & 35 \\
\hline 1934 & 3.982795 & -8.23895 & 6.447109 & 3.272446 & 11.7926 & 17.05317 & 2.634 & 36 \\
\hline
\end{tabular}




\begin{tabular}{|c|c|c|c|c|c|c|c|c|}
\hline 1949 & 3.969248 & -8.29151 & 6.50847 & 3.310986 & 11.85032 & 17.15423 & 2.634 & 37 \\
\hline 2005 & 3.963369 & -8.2032 & 6.418088 & 3.252412 & 11.71281 & 16.91381 & 2.633 & 38 \\
\hline 1947 & 3.961196 & -8.25633 & 6.496668 & 3.300623 & 11.8421 & 17.13525 & 2.618 & 39 \\
\hline 1983 & 3.942828 & -8.25215 & 6.48484 & 3.29192 & 11.78705 & 17.0612 & 615 & 40 \\
\hline 1954 & 3.94202 & -8.16981 & 6.393387 & 3.244238 & 11.67373 & 16.88136 & 2.608 & 41 \\
\hline 1994 & 3.929615 & -8.23147 & 6.465072 & 3.286152 & 11.75578 & 17.00483 & 2.605 & 42 \\
\hline 1977 & 3.925433 & -8.05524 & 6.302699 & 3.179317 & 11.3804 & 16.49163 & 2.569 & 43 \\
\hline 1996 & 3.881433 & -8.02709 & 6.267735 & 3.166216 & 11.35729 & 16.4135 & 2.558 & 44 \\
\hline 1963 & 3.879425 & -8.07637 & 6.322384 & 3.203014 & 11.47917 & 16.57627 & 2.551 & 45 \\
\hline 1992 & 3.877836 & -8.02063 & 6.255938 & 3.182188 & 11.41601 & 16.48086 & 2.547 & 46 \\
\hline 1995 & 3.86972 & -8.03878 & 6.283142 & 3.18044 & 11.41713 & 16.48211 & 2.546 & 47 \\
\hline 1979 & 3.865806 & -8.04053 & 6.294495 & 3.18636 & 11.41509 & 16.47804 & 2.544 & 48 \\
\hline 1951 & 3.845849 & -7.95636 & 6.214164 & 3.135211 & 11.24216 & 16.23212 & 2.540 & 49 \\
\hline 1997 & 3.844208 & -8.05372 & 6.319254 & 3.202019 & 11.43375 & 16.51276 & 2.525 & 50 \\
\hline 1971 & 3.838855 & -7.91574 & 6.193847 & 3.135487 & 11.26491 & 16.27181 & 2.524 & 51 \\
\hline 1978 & 3.823466 & -7.96203 & 6.247162 & 3.170972 & 11.3238 & 16.35059 & 2.519 & 52 \\
\hline 1975 & 3.772341 & -7.83217 & 6.110679 & 3.082703 & 11.01468 & 15.87567 & 2.464 & 53 \\
\hline 1976 & 3.755126 & -7.79189 & 6.088791 & 3.069454 & 10.93845 & 15.79496 & 2.452 & 54 \\
\hline 1974 & 3.71 & & 6. & 97 & 95042 & 15 & 2.435 & 55 \\
\hline 1973 & 3.701735 & -7.75512 & 6.060881 & 3.063336 & 10.88844 & 15.68703 & 2.423 & 56 \\
\hline 1970 & 3.677573 & -7.70947 & 6.035923 & 3.05188 & 10.82081 & 15.59016 & 2.409 & 57 \\
\hline 1957 & 3.659814 & -7.68056 & 6.008265 & 3.03068 & 10.75799 & 15.48909 & 2.393 & 58 \\
\hline 1972 & 3.620107 & -7.61571 & 5.956285 & 3.009259 & 10.65492 & 15.33504 & 2.367 & 59 \\
\hline 1950 & 3.56955 & -7.4756 & 5.865539 & 2.907946 & 10.49566 & 15.14866 & 2.337 & 60 \\
\hline 1965 & 3.555612 & -7.49582 & 5.853291 & 2.95037 & 10.4289 & 15.00284 & 2.316 & 61 \\
\hline 1968 & 3.473366 & -7.29213 & 5.686112 & 2.856599 & 10.04583 & 14.44506 & 2.277 & 62 \\
\hline 1961 & 3.455395 & -7.31377 & 5.716101 & 2.870219 & 10.10658 & 14.50721 & 2.244 & 63 \\
\hline 1958 & 3.448371 & -7.29522 & 5.743725 & 2.908215 & 10.27138 & 14.86336 & 2.242 & 64 \\
\hline 1960 & 3.443321 & -7.28738 & 5.690758 & 2.85632 & 10.06019 & 14.43522 & 2.232 & 65 \\
\hline 1933 & 3.44021 & -7.26985 & 5.680508 & 2.85143 & 10.03667 & 14.39776 & 2.230 & 66 \\
\hline 1948 & 3.432887 & -7.25711 & 5.670356 & 2.846259 & 10.01652 & 14.36421 & 2.224 & 67 \\
\hline 1964 & 3.431035 & -7.24787 & 5.658146 & 2.846155 & 10.00246 & 14.33896 & 2.222 & 68 \\
\hline 1945 & 3.426377 & -7.24317 & 5.660667 & 2.83876 & 9.992328 & 14.32875 & 2.219 & 69 \\
\hline 1962 & 3.41329 & -7.21923 & 5.636199 & 2.831483 & 9.948574 & 14.26059 & 2.209 & 70 \\
\hline 1936 & 3.412416 & -7.21813 & 5.630371 & 2.825652 & 9.930939 & 14.25011 & 2.206 & 71 \\
\hline 1956 & 3.405262 & -7.20874 & 5.644031 & 2.822356 & 9.931835 & 14.25194 & 2.206 & 72 \\
\hline 1967 & 3.349235 & -7.06397 & 5.514002 & 2.75762 & 9.702004 & 13.90095 & 2.160 & 73 \\
\hline 1953 & 3.293649 & -6.9902 & 5.470127 & 2.739002 & 9.570284 & 13.68923 & 2.125 & 74 \\
\hline 1952 & $*$ & $*$ & $*$ & $*$ & $*$ & $*$ & $*$ & \\
\hline 1955 & $*$ & $*$ & $*$ & $*$ & $*$ & $*$ & $*$ & \\
\hline
\end{tabular}

\footnotetext{
* represents the influenza A virus PA protein sequence data missing.
} 


\section{Discussion}

From Table 2, we can see that the CIVs in 2009, 1969 and 1959 have the highest ranking. Also the CIVs in 1993, 1991 are very high.

During 1957 to 1959, the Asian flu pandemic caused two million people's death all over the world, and it is one of the most serious outbreak in the history. The CIV in 1959 is significant bigger than that in nearby several years. The CIV in 1954 is 2.608 , the CIV in1956 is 2.206, the CIV in 1957 is 2.393 , the CIV in 1958 is $2.242,1959$ is 5.684 , the CIV in 1960 is 2.232, the CIV in 1961 is 2.244 .

Hong Kong flu in 1969 is similar to the Asian flu in 1959. Maybe the affected people had accumulated related antibody in the Asian flu, so the Hong Kong flu had relatively fewer deaths than other epidemic. Estimated that there were 750,000 people died (in America 34,000 people died). And Hong Kong flu caused more than one million deaths. The CIV in 1969 is 8.383 , which is higher than $2.160,2.277,2.409,2.524$ in the nearby years.

In 2009 there was well-known serious bird flu. The CIV in 2009 is 30.274 which is the highest in all years, and it is higher than 5.135, 2.524, 4.741, 4.661 in nearby years .

During 1986 to 1993, there are a lot of human infected the swine flu epidemic in many areas of the world. From Table 2 we can see the CIV in these years are bigger than that in near years.

It is found that those influenza A virus pandemic years are almost at the top of the list, so we can verify those influenza A virus pandemic years based on the model, and draw a conclusion that there maybe an influenza A virus pandemic when the CIV in the year is significantly bigger than that in the nearby years and the CIV in the year is more than 3 (Fig. 1). Therefore we can regard the principal component model as an early-warning model to predict the pandemic year, and also verify its validity and objectivity. In this way we can take prevention and control.

In this paper, we might have not choose the best indexes that can completely reflect the protein data information of influenza A virus, so we should make further research and improvement in the future work. In addition, due to the lack of partial year data (e.g.1952, 1955), we cannot calculate the CIVs of these mentioned years, which it will affect our prediction and analysis of the pandemic years.

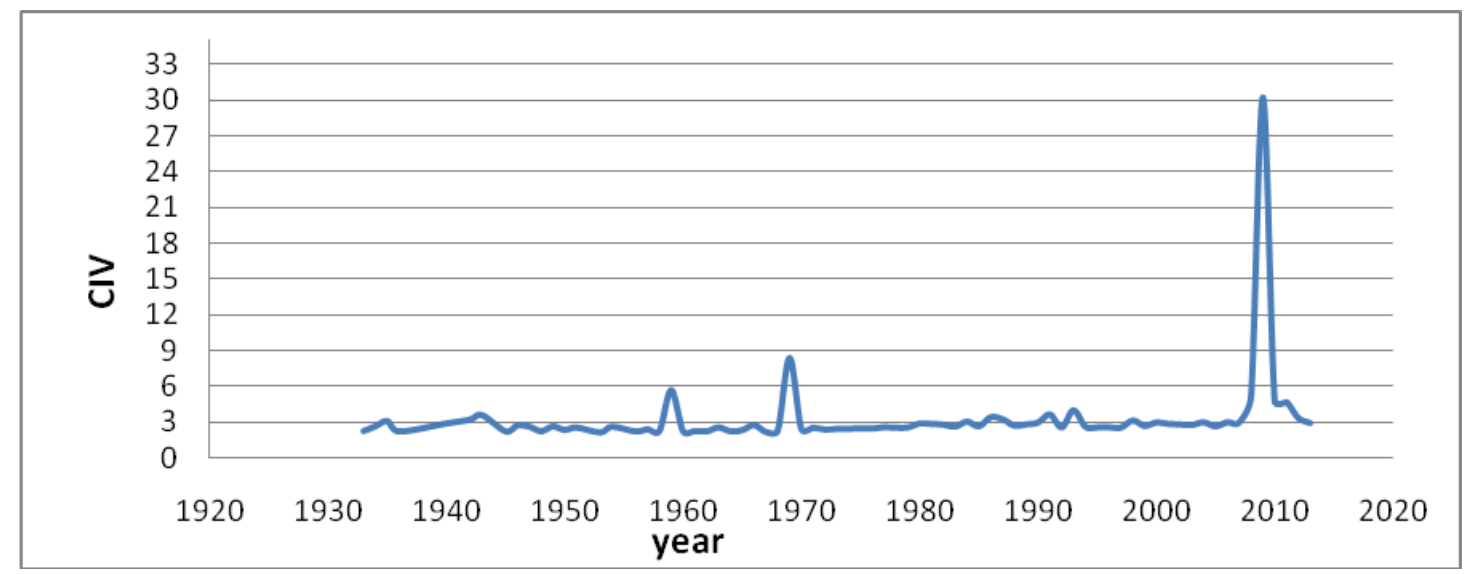

Figure 1. The CIV data from 1933 to 2013 
Acknowledgments. The project was supported by the National Natural Science Foundations of China (Grant No. 11271163 and No. 11371174).

\section{REFERENCES}

[1] Basu, S., Pan, A., Dutta, C., Das, J. (1997): Chaos game representation of proteins.-J. Mol. Graph. Model 15: 279-289.

[2] Fisher, A., Tusnady, G.E., Simon, I. (1994): Chaos game representation of protein structures.-J. Mol. Graphics 12: 302-304.

[3] Gao, J., Xu, Z.Y. (2009): Chaos game representation walk model for the protein sequences.-Chin. Phys. B 18: 4571-4579.

[4] Gao, R.B., Cao, B., Hu, Y.W., Feng, Z.J., Shu, Y.L., et al. (2013): Human infection with a novel Avian-Origin influenza A(H7N9) Virus.-N Engl J Med 368: 1888 -1897.

[5] Jeffrey, H. J. (1990): Chaos game representation of gene structure.-Nucleic Acid Res 18: 2163-2170.

[6] Johnson, R. A., Wichern, D. W. (2001): Applied multivariate statistical analysis. -Tsinghua Press, Beijing, 388. [in Chinese]

[7] Kobasa, D., Takada, A., Shinya, K., et al. (2004): Enhanced virulence of influenza A viruses with the haemagglutinin of the 1918 pandemic virus. -Nature 431: 703-707.

[8] Morens, D., Folkers, G., Fauci, A. (2004): The challenge of emerging and re-emerging infectious disease.-Nature 463: 242-249.

[9] Pu, J., Wang, S., Yin, Y., Zhang, G., Carter, R.A., et al. (2015): Evolution of the H9N2 influenza genotypes that facilitated the genesis of the novel H7N9 virus.-PNAS 112: 548-553.

[10] Ren, D., Gao, J. (2011): Early-warning signals for an outbreak of the influenza pandemic.-Chin. Phys. B 12: 128701.

[11] Scheffer, M., Bascompte, J., Brock, W. A., et al. (2009): Early-warning signals for critical transitions.-Nature 461: 53-59.

[12] Sokolov, D. N., Zarubaev, V. V., Shtro, A. A., et al. (2012): Anti-viral activity of (-)- and (+)-usnic acids and their derivatives against influenza virus A(H1N1)2009.-Bioorganic \& Medicinal Chemistry Letters 22: 7060-7064.

[13] Yu, Z.G., Anh, V.V., Lau, K.S. (2003): Multifractal and correlation analysis of protein sequences from complete genome.-Phys. Rev. E 68: 021913.

[14] Yu, Z.G., Anh, V.V., Lau, K.S. (2010): Chaos game representation of protein sequences based on the detailed HP model and their multifractal and correlation analyses.-J. Theor. Biol 226: 341-348. 